\title{
铑磑催化剂催化甲醇羰基化反应的 IRC 解析*
}

\author{
郝茂荣 ${ }^{(1)}$ 冯文林 ${ }^{(\mathbb{*} * *}$ 冀永强 $^{(2)}$ 雷 鸣 ${ }^{(1)}$
}

(1)北京化工大学理学院, 北京 100029; (2)宁夏大学化学化工学院, 银川 750021)

\begin{abstract}
摘要在 HF/LANL2DZ 水平上, 采用能量梯度法, 研究了铑碘催化剂催化甲醇羰基 化反应循环中各基元反应的反应物、中间体、过渡态、产物的几何结构，并计算了它们 势能面的变化. 通过对各基元反应过渡态的 IRC 解析, 证实了所得各类几何结构是甲 醇羰基化铑碘催化循环反应途径上的驻点, 完整给出了循环反应过程中分子沿极小能 量途径在各基元反应过程中的构型变化, 并提出了中间体构型转换在循环反应过程中 的作用。通过结构分析, 提出了顺式和反式催化循环反应两种途径之间除催化活性物 顺反异构关联外, 还可以通过中间体构型转换关联, 其活化能为 $49.79 \mathrm{~kJ} / \mathrm{mol}$, 并且在 还原消除基元反应步骤有既非顺式也非反式的情况，证明该循环反应可能经历多个途 径实现, 但无论何种途径, 碘甲烷氧化加成基元反应是整个循环反应过程的速控步骤.
\end{abstract}

\section{关键词 铑碘配合物催化剂 甲醇羰基化 从头算法 均相催化}

甲醇羰基化制乙酸是 60 年代末开发出来的乙酸制备工艺技术上最先进的工业方法, 鉴于 该法中多采用可溶性的羰基铑碘配合物做催化剂 ${ }^{[1,2]}$, Forster 在对有关反应中间体红外光谱分 析的基础上，提出了由 $\mathrm{CH}_{3} \mathrm{I}$ 氧化加成、羰基插人、 $\mathrm{CO}$ 配位和 $\mathrm{CH}_{3} \mathrm{COI}$ 还原消除基元反应构 成的循环反应机理 ${ }^{[3]}$, 并认为催化活性物是顺式 $\left(\mathrm{Rh}(\mathrm{CO})_{2} \mathrm{I}_{2}\right)^{-}$. 这种推测在实验研究上还不能 完全确认，人们又一直没有从分子反应动态学的理论上对该催化循环反应进行系统的研究. 最近, 雷鸣等 ${ }^{[4] 1)}$ 对该催化循环反应进行了较为系统的量子化学理论研究, 给出了反式或顺式 $\left[\mathrm{Rh}(\mathrm{CO})_{2} \mathrm{I}_{2}\right]^{-}$催化的这种循环反应中每个基元反应的中间体、过渡态的几何构型和反应活化能, 理论上描述了两个各自独立的催化循环途径. 为了从理论上确认这些驻点的合理性, 并探讨 顺式和反式催化循环之间的关联性, 本文依据内禀反应坐标法(IRC)理论, 分别对顺式和反式 铑碘配合物催化甲醇羰基化反应循环机理的各个基元反应进行 IRC 解析, 为进一步认识该催 化反应机理提供理论依据.

\section{1 计算方法}

本文采用有效核势能近似(ECP)从头算方法, 在 HF/LANL2DZ 水平上, 运用能量梯度法优 化了甲醇羰基化铑碘催化循环中反应的反应物、中间体、过渡态、产物的几何结构. 通过对所 涉及的过渡态结构进行振动分析, 得出的惟一虚振动频率, 确认了过渡态结构. 在此基础上, 对各反应过渡态进行了 IRC 解析, 获得了各基元反应中反应分子结构变化规律. 全部计算工

2003-01-28 收稿, 2003-07-21 收修改稿

* 国家教委博士点基金资助课题(批准号: 1999902735)

***联系人, E-mail: fengwl@buct.edu.cn

1) 雷 鸣, 等. 顺式铑碘配合物催化甲醇羰基化反应机理的从头算研究 
作采用 GAUSSIAN-98 量子化学计算程序完成 ${ }^{[5]}$.

\section{2 结果和讨论}

反式铑碘配合物催化甲醇羰基化反应循环的反应物、中间体、过渡态和产物结构见图 1 . 循环反应包括了三个基元反应, 即 $\mathrm{CH}_{3} \mathrm{I}$ 氧化加成基元反应：( $\left.\mathrm{Rh}(\mathrm{CO})_{2} \mathrm{I}_{2}\right)^{-}$和 $\mathrm{CH}_{3} \mathrm{I}$ 经过渡态 TS-1A，到中间体 A2; 羰基插人基元反应： A2 经过渡态 TS-2A 到中间体 A3；还原消除基元反 应：A4 经过渡态 TS-3A 到产物. 本文在对上面基元反应的过渡态做 IRC 解析时, 发现过渡态 TS-2A 所联系的产物 A3 结构不同于前文 ${ }^{[4]}$ 给出的结构. 进一步研究指出, 在联系 A3 和 A4 两 个结构之间还存在两个新的构型过渡态 ROT-TS1 和 ROT-TS2, 对其进行 IRC 解析找到另两个 中间体 ROT-INT1 和 ROT-INT2. CO 和 ROT-INT2 配位生成 A4, 是无位垒过程. 以上各构型的 结构参数见图 1 .

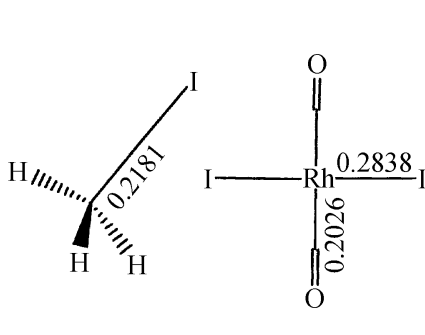

$\mathrm{CH} 3 \mathrm{I}$

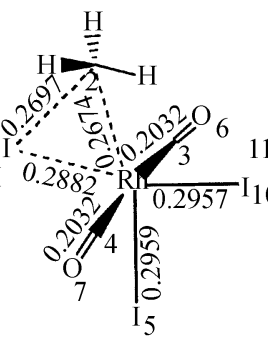

TS-1A

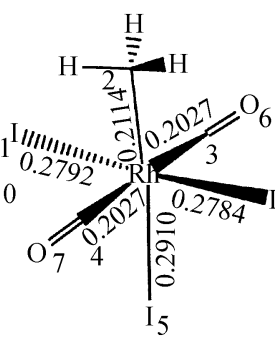

A2

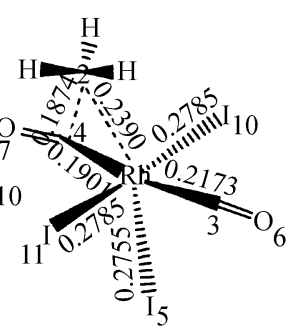

TS-2A
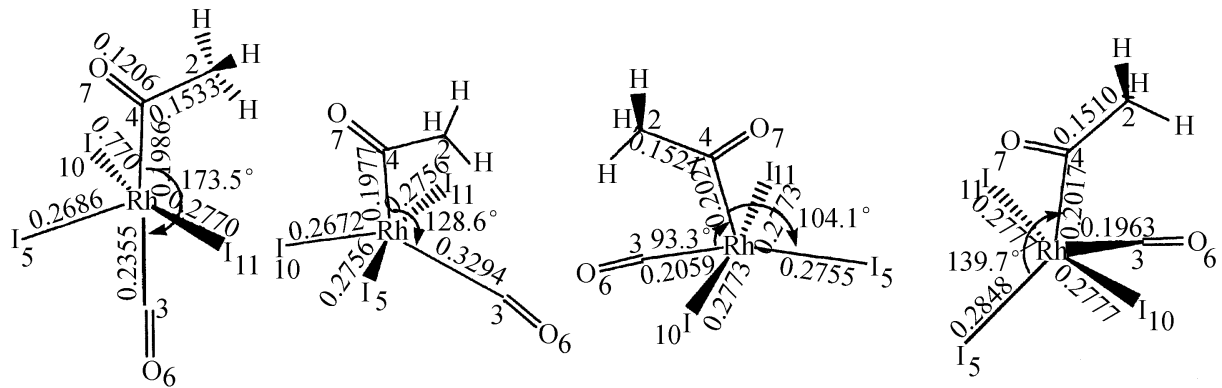

A3

ROT-TS 1

ROT-INT1

ROT-TS 2

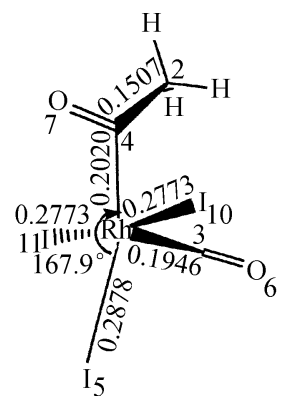

ROT-INT2

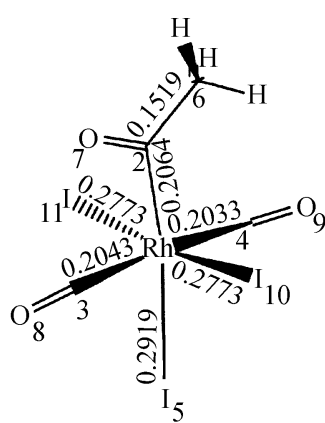

A4

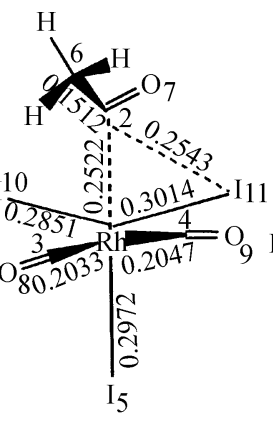

TS-3A

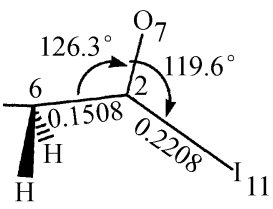

$\mathrm{CH}_{3} \mathrm{COI}$

图 1 甲醇崤基化催化反应反式循环中反应物、中间体、过渡态和产物的几何构型

顺式铑碘配合物催化甲醇羰基化反应循环的反应物、中间体、过渡态和产物结构见图 2 . 


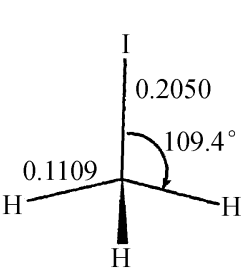

$\mathrm{CH}_{3} \mathrm{I}$

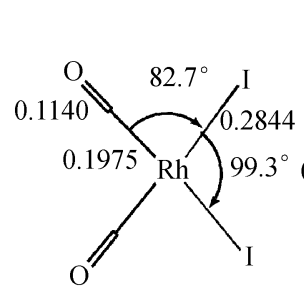

$\left[\mathrm{Rh}\left(\mathrm{CO}_{2}\right) \mathrm{I}_{2}\right]^{-}$

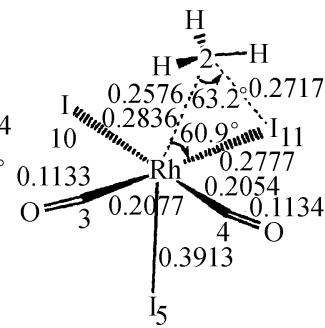

TS-1B

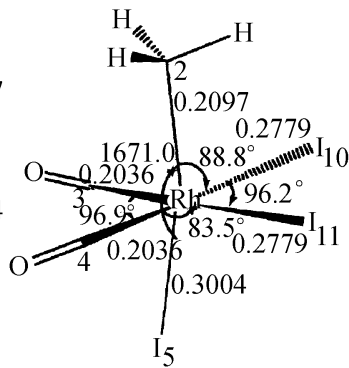

B2

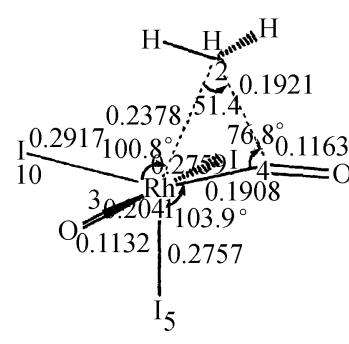

TS-2B

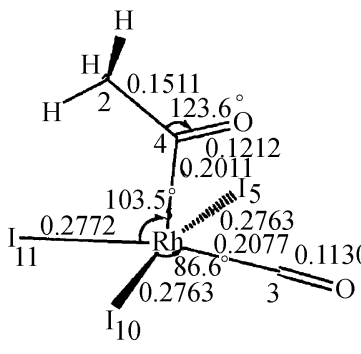

B3

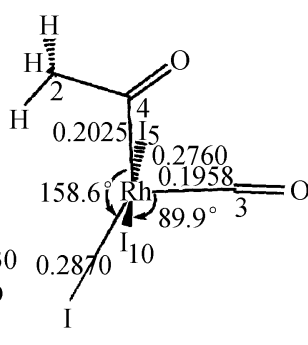

ROT-TS3

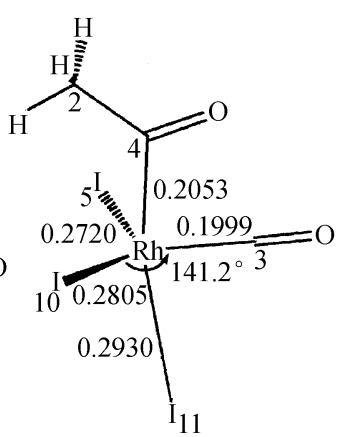

ROT-INT3

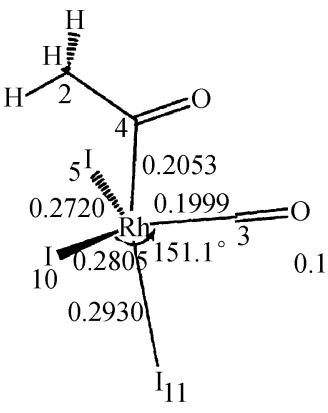

ROT-INT 4

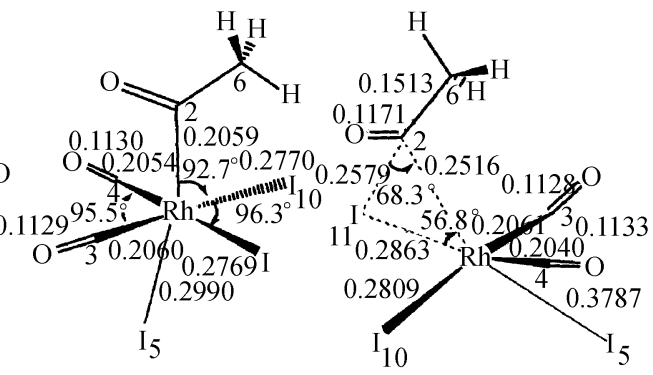

B4

TS-3B

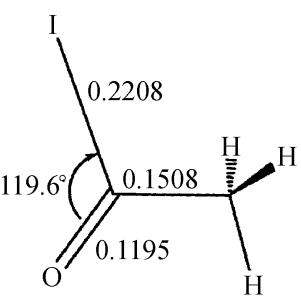

$\mathrm{CH}_{3} \mathrm{COI}$

图 2 甲醇羰基化催化反应顺式循环中反应物、中间体、过渡态和产物的几何构型

循环反应中, $\mathrm{CH}_{3} \mathrm{I}$ 氧化加成基元反应: $\left(\mathrm{Rh}(\mathrm{CO})_{2} \mathrm{I}_{2}\right)^{-}$与 $\mathrm{CH}_{3} \mathrm{I}$ 经过渡态 $\mathrm{TS}-1 \mathrm{~B}$ 到中间体 $\mathrm{B} 2$; 羰基插人基元反应： B2 经过渡态 TS-2B 到中间体 B3; 还原消除基元反应： B4 经过渡态 TS-3B 到产物. 本文在对上述基元反应的过渡态做 IRC 解析时, 得到的 B3 并不能和 CO 直接配位生 成 B4 构型. 研究认为在羰基配位基元反应步骤之前还存在两步构型转变, 并找到了这两个构 型转变的过渡态 ROT-TS3 和 ROT-TS4, 以及两个新的中间体 ROT-INT3 和 ROT-INT4. 羰基 配位基元反应是 ROT-INT4 和 CO 反应生成 B4，配位过程无位垒.

比较顺、反式铑碘配合物催化甲醇羰基化反应循环的情况, 顺式循环中的 B3 结构和反式 循环中的 ROT-INT1 结构极其相似. 研究发现, 这两个结构之间可通过过渡态 TS-AB(虚振动 频-70.7 $\mathrm{icm}^{-1}$ )进行关联, 其结构见图 3, 主要是乙酰基的空间位置不同, 介于 B3 和 ROT-INT1 
结构之间, 这就说明, 顺式循环和反式循环反应可通过这一过渡态实现构型转变而相互关联; 此外, 在不考虑循环过程保持顺式或反式结构的情况下, CO 可直接与 B3 和 ROT-INT1 进行羰 基配位反应, 即 $\mathrm{B} 3+\mathrm{CO} \rightarrow \mathrm{C}$, ROT-INT $1+\mathrm{CO} \rightarrow \mathrm{D}$, 是无位垒过程, $\mathrm{C}$ 和 $\mathrm{D}$ 的结构见图 3 , 进 一步发生还原消除基元反应, 即 $\mathrm{C}$ 经过渡态 C-TS(振动分析有惟一负频 $-238.7 \mathrm{icm}^{-1}$ )到产物, D 经过渡态 D-TS(振动分析有惟一负频 $-225.8 \mathrm{icm}^{-1}$ ) 到产物, 以上构型的结构参数见图 3 .
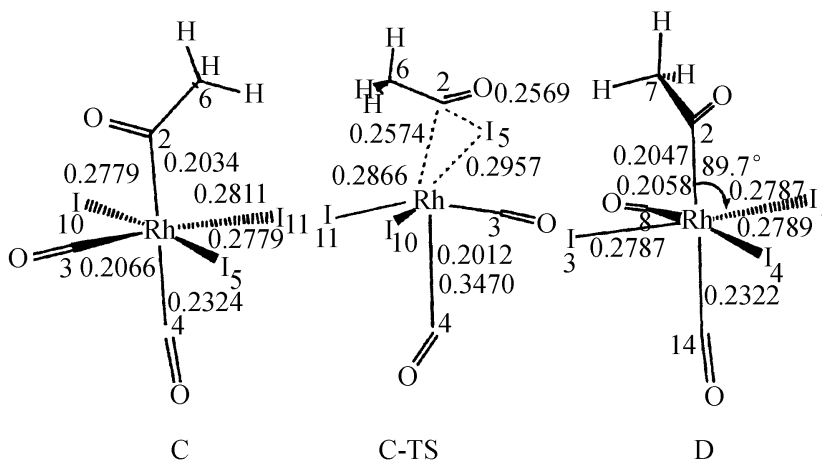

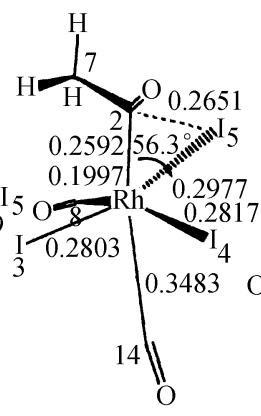

D-TS

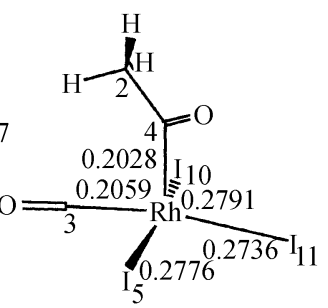

TS-AB

图 3 甲醇羰基化催化反应循环中其他过程的反应物、中间体、过渡态和产物的几何构型

甲醇羰基化循环反应有如图 4 所示的可能途径. 以下按不同基元反应步骤，分别讨论顺、 反式铑碘配合物催化反应的 IRC 解析结果.

\section{1 碘甲烷氧化加成基元反应的 IRC 解析}

2.1.1 反式过程 通过对这一基元反应的 IRC 解析, $\mathrm{C}(2)-\mathrm{Rh}, \mathrm{C}(2)-\mathrm{I}(11)$ 以及 $\mathrm{I}(11)-\mathrm{Rh}$ 等键的原子间距离变化随反应进程的关系见图 5. 反应开始, $\mathrm{C}(2)-\mathrm{Rh}$ 原子间距离就急剧缩短, 直到 $S=5$ 以后变化平缓; 而 $\mathrm{C}(2)-\mathrm{I}(11)$ 原子间距离的变化开始时不大, 在 $S=-4$ 后却开始急 剧伸长; I(11)一 Rh 原子间距离缩短的趋势相对小一些, 特别是过渡态之后, 原子间距离缩短的 趋势变的平缓. 这说明该反应过程, 首先是 $\mathrm{CH}_{3} \mathrm{I}$ 沿 $\mathrm{C}(3) \mathrm{O}-\mathrm{Rh}-\mathrm{C}(4) \mathrm{O}$ 键垂直的平面方向整 体向金属中心 $\mathrm{Rh}$ 靠近, 此时原子间距离变化程度依次为 $\mathrm{C}(2)-\mathrm{Rh}>\mathrm{I}(11)-\mathrm{Rh}>\mathrm{C}(2)-\mathrm{I}(11)$, $\mathrm{C}(2)$ 和 $\mathrm{I}(11)$ 分别与 $\mathrm{Rh}$ 逐步成键, 到一定程度(约 $S=-4)$ 之后, $\mathrm{C}(2)-\mathrm{Rh}$ 键的形成和 $\mathrm{C}(2)-\mathrm{I}(11)$ 键的断裂几乎同步同程度进行, 而 $\mathrm{I}(11)-\mathrm{Rh}$ 原子间距离变化很缓慢, 接近成键. 从构型上看, 随反应进行, $\mathrm{C}(2)$ 摆到四角双雉的雉顶点, 同时伴随着 $\mathrm{I}(5)$ 也摆到另一个雉顶点, I(5)- $\mathrm{Rh}$ 原子 间距离由于排斥作用略有伸长.

\subsection{2 顺式过程 顺式过程与反式过程相似, 见图 6, 反应一开始, $\mathrm{C}(2)-\mathrm{Rh}$ 原子间距离就} 急剧缩短, 而其他的键长基本不变, 在过渡态前后 $(-1.5<S<1.5), \mathrm{C}(2)-\mathrm{Rh}$ 键的形成和 $\mathrm{I}(11)-$ $\mathrm{C}(2)$ 键的断裂几乎是同步同程度进行的. 不同的是在反应过程中 $\mathrm{CH}_{3} \mathrm{I}$ 向催化剂平面接近时, 由于 $\mathrm{CH}_{3} \mathrm{I}$ 分子中的 $\mathrm{I}$ 和中心原子 $\mathrm{Rh}$ 成键时对原有配位的 $\mathrm{I}$ 的排斥作用, 造成构型畸变.

\section{2 羰基插入基元反应的 IRC 解析}

2.2.1 反式过程 对湠基插人基元反应进行 IRC 解析, 从图 7 看出, $\mathrm{C}(2)-\mathrm{C}(4)$ 原子间距离 随反应进程急剧缩短, 到达过渡态后已很快成键; $\mathrm{C}(2)-\mathrm{Rh}$ 原子间距离在过渡态附近 $(S=$ -0.5 0.5)变化平缓, 此后变化加剧; $\mathrm{C}(4)$ - $\mathrm{Rh}$ 原子间距离变化不大, 特别是 $S=-0.5 \sim 0.5$ 内基 


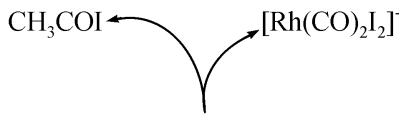

TS-3B

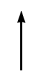

B4

$\mathrm{CO}+\uparrow$

ROT-INT4

$\uparrow$

ROT-TS4

$\uparrow$

ROT-INT3

$\uparrow$

ROT-TS3

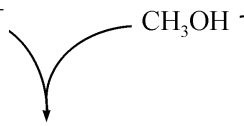

TS-1B

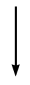

B2

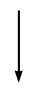

TS-2B
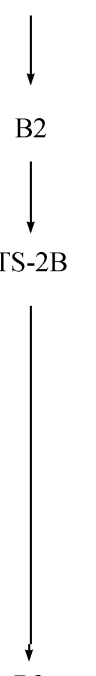

2

TS-AB

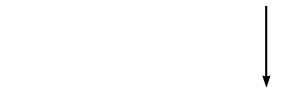

A3

TS-1A

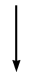

A2

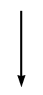

TS-2A

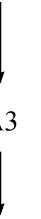

ROT-TS1
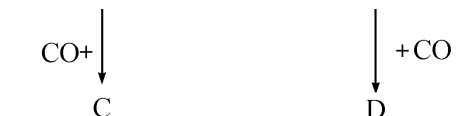

$\left[\mathrm{Rh}(\mathrm{CO})_{2} \mathrm{I}_{2}\right]^{-}+\mathrm{CH}_{3} \mathrm{COI}$

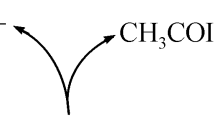

TS-3A

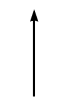

A4

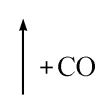

ROT-INT2

图 4 甲醇羰基化反应循环机理

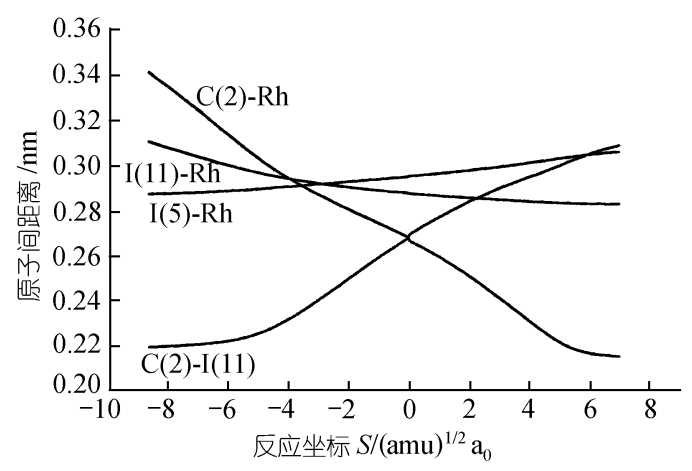

图 5 碘甲烷氧化加成基元反应原子间距离随 反应进程的变化

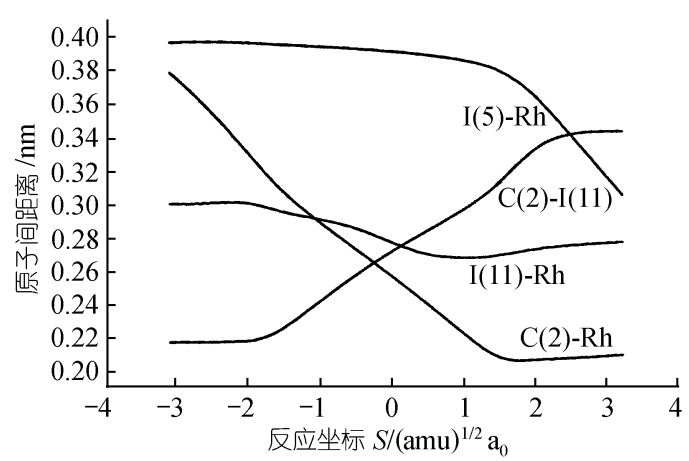

图 6 碘甲烷氧化加成基元反应原子间距随 反应进程的变化

本不变, 只是构型调整中因受到其他配体的排斥而略有变化. 显然, 反应开始时甲基 $\mathrm{C}(2)$ 向羰 基 $\mathrm{C}(4)$ 靠拢, 甲基迁移, 适当拉长 $\mathrm{C}(2)-\mathrm{Rh}$ 键, 较迅速地生成 $\mathrm{C}(2)-\mathrm{C}(4)$ 键, 待 $\mathrm{C}(2)-\mathrm{C}(4)$ 
键基本形成后, $\mathrm{C}(2)-\mathrm{Rh}$ 键快速断裂，所经过的过渡态是类产物型的.

2.2.2 顺式过程图 8 给出了反应中原子间距随反应进程的变化, 看得出类似反式的情况, 反应过程实质上是甲基向羰基迁移, 待 $\mathrm{C}(2)-\mathrm{C}(4)$ 键基本形成后, $\mathrm{C}(2)-\mathrm{Rh}$ 键迅速断裂, 完成 反应.

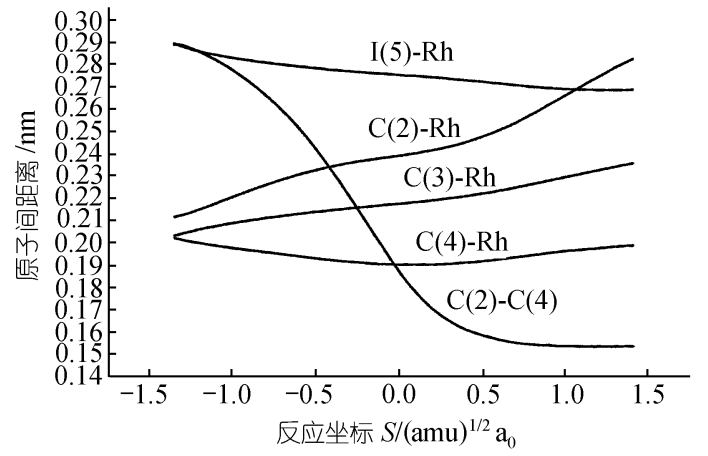

图 7 羰基插人基元反应原子间距随反应 进程的变化

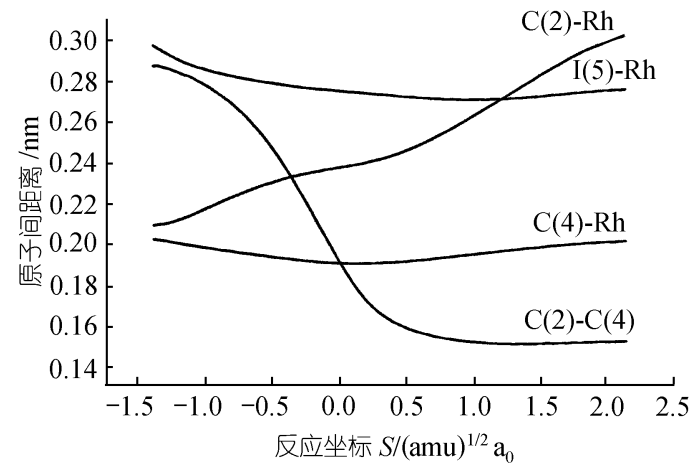

图 8 羰基插人基元反应原子间距随反应 进程的变化

通过对羰基插人反应进行 IRC 解析，结果都表明该反应的实质是甲基迁移.

\subsection{CO 配位基元反应的 IRC 解析}

\subsection{1 反式过程 根据羰基插人基元反应的 IRC 解析结果和还原消除基元反应的 IRC 解析} 结果的对比分析, A3 和 A4 两者构型上存在着差异,要求 A3 与 CO 配位时其构型必须要调整. 本文研究指出, 由 A3 到 A4 构型是经过两个构型转变过渡态, 即 ROT-TS1 和 ROT-TS2 来完成. 对两个构型转变过渡态进行 IRC 解析后, 得到反应中间体 ROT-INT1 和 ROT-INT2(结构见图 1). 在 A3 经 ROT-TS1 到 ROT-INT1 的过程中, 主要是 键角 $\angle \mathrm{C}(3) \mathrm{Rh} C(4)$ 由 $173.5^{\circ}$ 变成 $93.3^{\circ}$, 其他有关键原子间距离变化很小. 在 ROT-INT1 经 ROT-TS2 到 ROT-INT2 的过程中, 主要是键角 $\angle \mathrm{I}(5) \mathrm{Rh}$ C(4)由 $104.1^{\circ}$ 变成 $167.9^{\circ}$.此后 CO 与 ROT-INT2 配位得到 A4.

2.3.2 顺式过程 依顺式循环中羰基插人基元反应的 IRC 解析和还原消除基元反应的 IRC 解析结果, 对比分析 B3 与 B4 构型上的差异, 需对 B3 构型进行转变. 我们的研究发现, 第一 步是 B3 结构中的 $\angle \mathrm{C}(4) \mathrm{RhI}(11)$ 经过渡态 ROT-TS3 由 $103.5^{\circ}$ 增大到 $172.0^{\circ}$, 得到中间体 ROT-INT3; 第二步是 ROT-INT3 结构中的 $\angle \mathrm{I}(10)$ Rh C(3)经过渡态 ROT-TS4 由 $91.9^{\circ}$ 增大到 $151.1^{\circ}$ ，得到中间体 ROT-INT4. 然后 ROT-INT4 和 CO 配位得到 B4.

\section{4 还原消除基元反应的 IRC 解析}

2.4.1 反式过程 对反应途径的 IRC 研究发现(见图 9), 在反应开始时 I(11)-C(2)和 $C(2)$ 一 $\mathrm{Rh}$ 原子间距离的变化明显, 前者缩短, 后者伸长, 而 $\mathrm{I}(11)-\mathrm{Rh}$ 原子间距离变化很缓慢, 直 到 $S=0.5$ 后, I(11)一 $\mathrm{Rh}$ 原子间距离急剧伸长. 这说明反应开始是 $\mathrm{C}(2)$ 向 $\mathrm{I}(11)$ 靠拢, 逐渐形成 $\mathrm{I}(11)-\mathrm{C}(2)$ 键, 而 $\mathrm{I}(11)$ 缓慢离开 $\mathrm{Rh}$, 直到近过渡态 $(S=0.5$ 以后)后, $\mathrm{I}(11)-\mathrm{Rh}$ 和 $\mathrm{C}(2)-\mathrm{Rh}$ 原 子间距离才急剧伸长, 并断键, 产物脱离.

2.4.2 顺式过程 该基元反应途径的 IRC 研究见图 10, 可以看出, 在反应开始时, I(11)- 


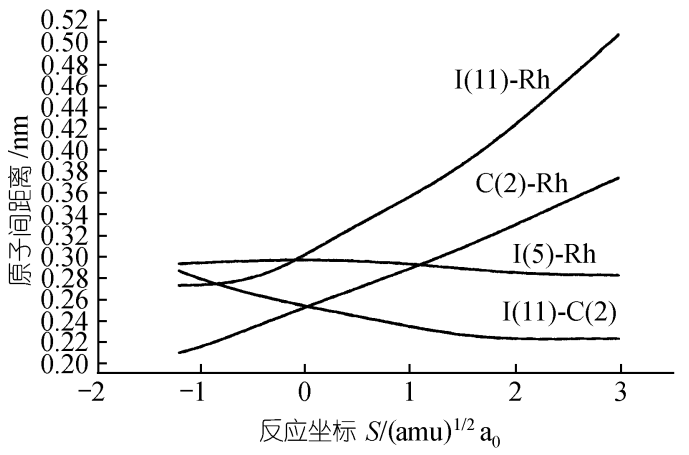

图 9 还原消除基元反应原子间距随反应 进程的变化

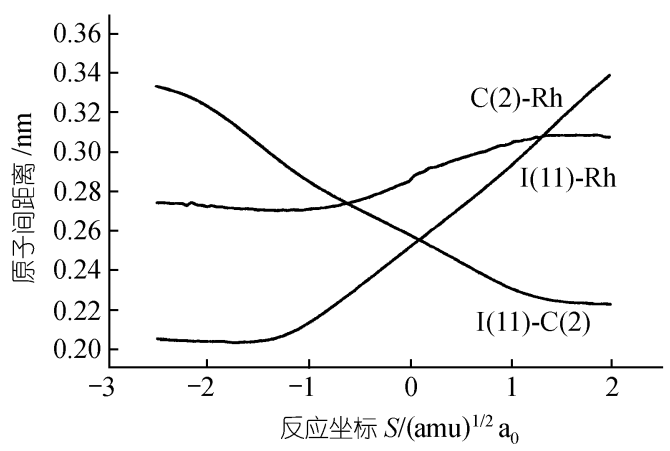

图 10 还原消除基元反应原子间距随反应 进程的变化

$\mathrm{C}(2)$ 原子间距离缩短, 变化显著, 而 $\mathrm{C}(2)-\mathrm{Rh}$ 和 $\mathrm{I}(11)-\mathrm{Rh}$ 原子间距离几乎不变, 说明 $\mathrm{I}(11)$ 与 $\mathrm{C}(2)$ 相向摆动, 互相靠近. 在 $S=-1.5 \sim 1.5$ 间, $\mathrm{C}(2)-\mathrm{Rh}$ 原子间距离伸长和 $\mathrm{I}(11)-\mathrm{C}(2)$ 原子 间距离缩短几乎同程度变化, 同时 $\mathrm{I}(11)-\mathrm{Rh}$ 原子间距离也不断地伸长, 最终产物脱离, 这是 不同于反式的.

\section{5 其他过程的 IRC 解析}

\subsection{1 顺式和反式循环的关联 以上研究表明, 铑碘催化剂催化甲醇羰基化反应可根据} 循环过程中羰基和 I 及中心原子 $\mathrm{Rh}$ 构成的平面的特点, 反应可分别沿顺式或反式路径进行. 但比较两个循环中 CO 配位基元反应前的情况，发现两个路径都经历了一个乙酰基在雉顶的 四角单锥稳定构型, 即顺式循环中的 B3 结构和反式循环中的 ROT-INT1 结构, 但两者结构上 只差乙酰基中酰氧基和甲基的位置对调. 本文研究证明, 在这两个结构之间存在一个联系两 者的构型转变过渡态 TS-AB(结构见图 3). 据此可以认为顺式和反式循环反应途径存在交叉.

\subsubsection{B3, ROT-INT1 结构和 CO 直接配位并发生 $\mathrm{CH}_{3} \mathrm{COI}$ 消除反应＼cjkstart在不考虑反应过} 程保持顺式或反式要求的情况下, 研究发现 B3 和 CO 经一无位垒过程直接配位生成一稳定结 构 C, ROT-INT1 也可和 CO 直接配位生成一稳定结构 D, C 和 D 的结构都是四角双雉结构, 但 $\mathrm{Rh}$ 作为中心原子的平面中不再存在顺式和反式 的结构特点, $\mathrm{C}$ 和 $\mathrm{D}$ 的结构差别也仅在于乙酰基 分子平面相对于 $\mathrm{Rh}$ 所在平面位置的不同. 进一 步研究表明, $\mathrm{C}$ 结构经过渡态 C-TS 发生 $\mathrm{CH}_{3} \mathrm{COI}$ 还原消除反应, 生成产物; D 结构经过渡态 D-TS 进行 $\mathrm{CH}_{3} \mathrm{COI}$ 还原消除反应, 生成产物.

以上研究使我们认识到, 铑碘催化剂催化 甲醇羰基化反应是一个存在多种途径的反应. 根据催化剂的结构特点, 反应可分别沿顺式路 径或反式路径进行, 也可根据反应途径中中间 体的结构特点, 或和 $\mathrm{CO}$ 直接配位完成反应, 或

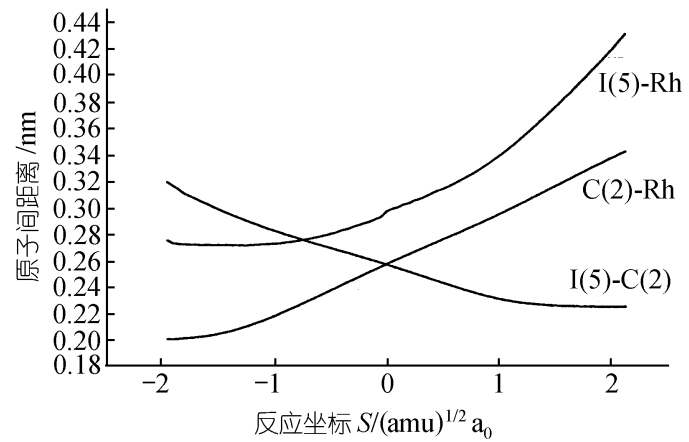

图 11 还原消除基元反应原子间距随反应进程 的变化 中间体发生构型转变等，使循环反应呈现途径的多样化特点. 见图 4.

图 11 是 C-TS 过渡态 IRC 解析结果, 表明了由 $\mathrm{C}$ 结构进行 $\mathrm{CH}_{3} \mathrm{COI}$ 消除反应过程中主要 
化学键沿反应途径的变化情况. 分析看出, 反应开始时 $\mathrm{I}(5)-\mathrm{C}(2)$ 原子间距离迅速缩短, 而 $\mathrm{I}(5)$ $-\mathrm{Rh}$ 和 $\mathrm{C}(2)-\mathrm{Rh}$ 原子间距离变化不大; 在 $S=-1 \sim 1$ 间 $\mathrm{I}(5)-\mathrm{C}(2)$ 键的形成和 $\mathrm{C}(2)-\mathrm{Rh}$ 键的 断开几乎同程度进行, 并且 $\mathrm{I}(5)-\mathrm{Rh}$ 原子间距离也迅速增长, 产物脱离, 这一特点类似顺式循 环中 $\mathrm{CH}_{3} \mathrm{COI}$ 消除反应的情况.

D-TS 过渡态的 IRC 解析结果分析表明，和 C-TS 的 IRC 结果完全相似.

\section{6甲醇羰基化铑磑催化循环反应的位能变化}

对循环反应的各反应物、产物、中间体和过渡态位能都做了零点能校正后, 绘出了顺式和 反式循环反应中各基元反应步骤的位能面图(见图 12,13), 比较看出, 反式和顺式循环反应中, $\mathrm{CH}_{3} \mathrm{I}$ 氧化加成反应的反应位垒分别是 212.88 和 $216.59 \mathrm{~kJ} / \mathrm{mol}$, 差别很小; 羊基插人反应的位 垒分别是 128.57 和 $114.85 \mathrm{~kJ} / \mathrm{mol}$, 两者的差别也不大; $\mathrm{CH}_{3} \mathrm{COI}$ 还原消除反应的位垒差别最大, 反式循环中该基元反应位垒是 $124.6 \mathrm{~kJ} / \mathrm{mol}$ ，该值比顺式的低 $37.79 \mathrm{~kJ} / \mathrm{mol}$.

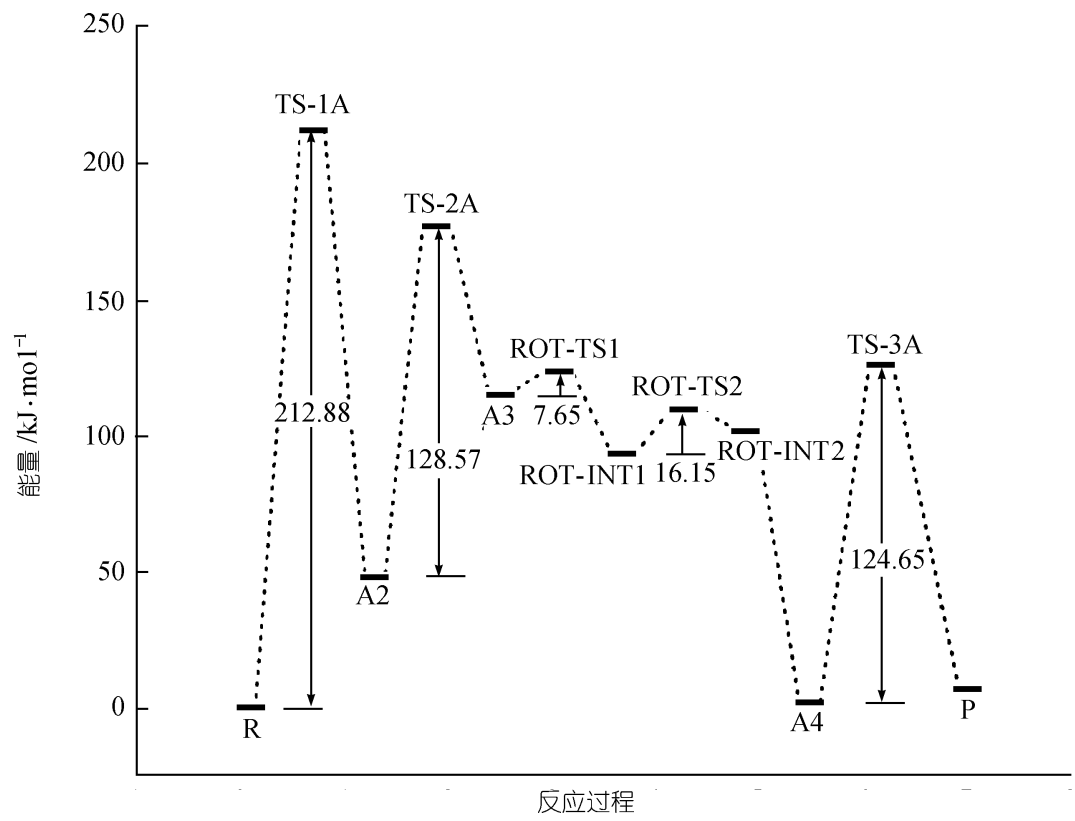

图 12 反式铑碘配合物催化甲醇嵈基化反应循环机理中各基元反应的位能

不计顺式和反式特征的另两个 $\mathrm{CH}_{3} \mathrm{COI}$ 还原消除反应步骤的反应位垒分别是 128.73 和 $113.81 \mathrm{~kJ} / \mathrm{mol}$ ，两者差别也不大，与反式循环中该基元反应的值相近.

关联顺式和反式循环的反应, 即 B3 经过渡态 TS-AB 到 ROT-int1 的反应位垒是 49.79 $\mathrm{kJ} / \mathrm{mol}$, 是一构型转变过渡态.

在 CO 配位前, 所发生的构型转变过程的位垒是顺式的要大于反式的. 无论是顺式循环反 应还是反式循环反应, 均以氧化加成步骤的位垒最高, 是整个循环反应过程的速控步骤.

\section{3 结论}

（1）各基元反应的 IRC 解析, 证明了优化所得到的中间体、过渡态是甲醇羰基化催化循环 反应的中间体、过渡态. 


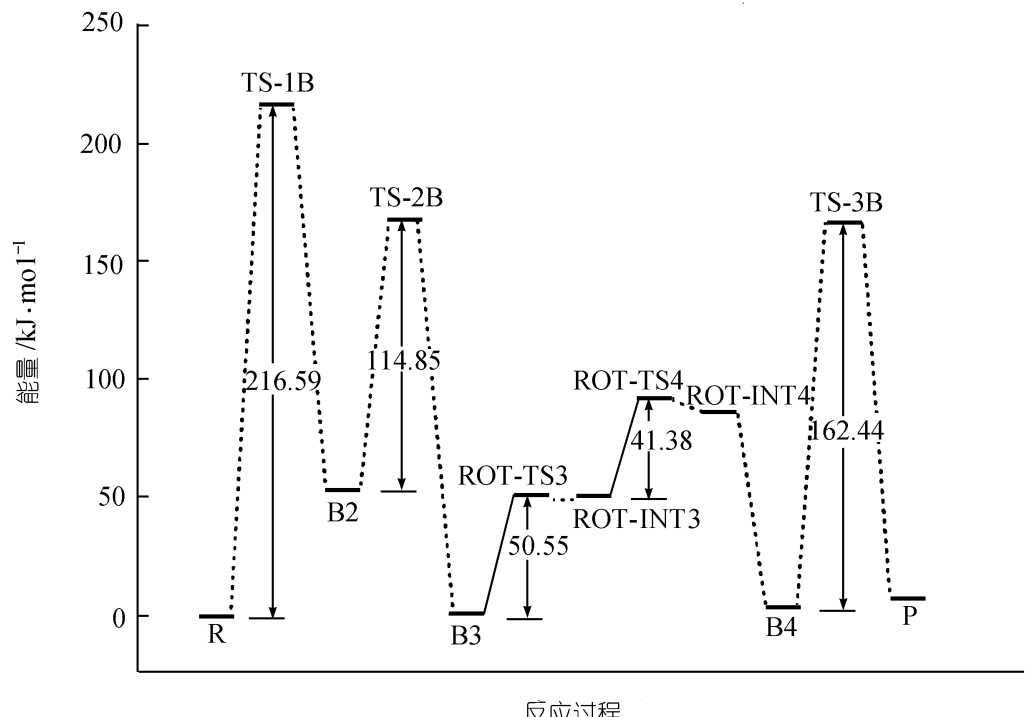

图 13 顺式铑碘配合物 $\left[\mathrm{Rh}(\mathrm{CO})_{2} \mathrm{I}_{2}\right]^{-}$催化甲醇羰基化反应的位能剖面

（2）经过 IRC 研究, 无论是顺式还是反式, 碘甲烷氧化加成和羰基插人基元反应的情况相 类似, 羰基插人反应的实质是由甲基迁移来实现的, 但是还原消除反应中产物脱离过程略有 不同; 要保持顺或反式结构特征, 在羰基插人和 CO 配位基元反应之间还存在构型转变过程, 该转变过程的位垒都不高, 但是顺式的要大于反式的.

（3）理论研究表明，甲醇羰基化循环反应可能经历多个途径实现. 除活性物的顺反式结 构互变外, 研究发现顺式和反式循环反应之间存在一个关联点, 证明了这两个循环不是各自 独立的，它们可以进行反应交叉和关联；在不考虑循环反应过程保持顺式或反式结构要求的 情况下, 羰基插人反应的产物可和 CO 直接发生配位反应, 进而发生还原消除反应, 生成产物 $\mathrm{CH}_{3} \mathrm{COI}$.

（4）比较循环反应的位能变化情况，顺式和反式循环反应中对应各步基元反应的位垒有 一定的差别, 主要是在还原消除反应步骤, 该步的位垒是反式的比顺式的低 $37.79 \mathrm{~kJ} / \mathrm{mol}$. 但 是, 无论对顺式循环还是对反式循环, 或其他途径, $\mathrm{CH}_{3} \mathrm{I}$ 氧化加成位垒最高, 是循环反应的速 控步骤，这与实验事实相符.

\section{参考文献}

1 Paulik F E, Roth J F. Catalysts for the low-pressure carbonylation of menthanol to acetic acid. Chem Commumn, 1968, 24: $1578 \sim 1581$

2 Jiang H, Diao K S, Pan P 1, et al. A new class of rhodium complexes containing free donor atoms and their intramolecular substitution reaction. Chin J Chem, 2000, 18: 752 755

3 Forster D. On the mechanism of a Rhodium complexex catalyzed methanol carbonylation of methanol to acetic acid. J Am Chem Soc, 1976, 98: 846 847

4 雷 鸣, 冯文林, 郝茂荣, 等. 甲醇羰基化制乙酸反应理论研究. 中国科学, B 辑, 2001, 31(5): 462 467

5 Frisch M J, Truncks G W, Schlegl H B, et al. Gaussian 98, Gaussian Inc, Pittsburgh PA, 1998 